\title{
SYNTHESYS OF A FUNCTIONALIZED FURAN FRAGMENT THROUGH BROMINATION-CYCLIZATION OF A CONJUGATED DIENE
}

\author{
Veaceslav Kulciţki ${ }^{1 *}$, Marina Cara ${ }^{1}$, Andrea Bourdelais², Tomas Schuster ${ }^{2}$ \\ and Daniel Baden ${ }^{2}$ \\ ${ }^{I}$ Institutul de Chimie al AŞ a RM,str. Academiei, 3, MD-2028, Chişinău, Republica Moldova \\ ${ }^{2}$ Center for Marine Sciences (CMS), University of North Carolina at Wilmington, 5600 Marvin K. Moss Lane, Wilmington, NC \\ 28409, USA \\ *kulcitki@yahoo.com, phone: +37322 739769,fax: +37322739769
}

\begin{abstract}
This paper describes the use of a terpenic model substrate to conjugate 1,4-additions of N-bromosuccinimide (NBS) to a diene system possessing a suitable oxygen functionality. The reaction leads to functionalized tetrahydrofuran derivatives, which can be developed further into different synthetic targets, such as polyethers, which are known to possess diverse biological activity.
\end{abstract}

Keywords: Terpenoids, furans, NBS, conjugate addition.

\section{Introduction}

Polyethers are widespread in natural sources and are known for biological activity related to their metal complexation capability [1]. Many groups of natural products, such as $C$-nucleosides, acetogenins, brevetoxins, and ionophore antibiotics, have oxygen heterocycles incorporated into their structure. The presence of such functionalities helps to determine the bioactivity of the compound. The limited availability from renewable raw material has led to the elaboration of different synthetic tools to access cyclic ethers of different ring size [2]. Specific ring functionalization is often a requirement for the biological activity of target structures and their elaboration requires manipulation of complex synthetic intermediates. In this paper we describe a method for the synthesis of functionalized cyclic ether moieties using a common terpene as a model compound.

\section{Results and discussion}

The range of synthetic procedures used to access functionalized $O$-heterocycles is quite broad. The most general approach is based on a biomimetic strategies and includes two successive operations:

1) assembling a structural fragment comprised of an olefinic double bond and a hydroxyl group in neighboring positions;

2) a C-O cyclizaton reaction initiated by different electrophilic, nucleophilic or radical agents [2].

Halo-etherification reactions are of special interest in this context, since they allow both cyclization and incorporation of a halogen atom in the cyclic structure. The halogen atom can subsequently be utilized as a synthetic handle. Normally, addition of the halogen occurs in a 1,2-fashion, and the generated carbocation cyclizes with a neighboring oxygen functionality to provide the corresponding cyclic ether (Scheme 1). This strategy works well for the synthesis of furan ring systems [2].
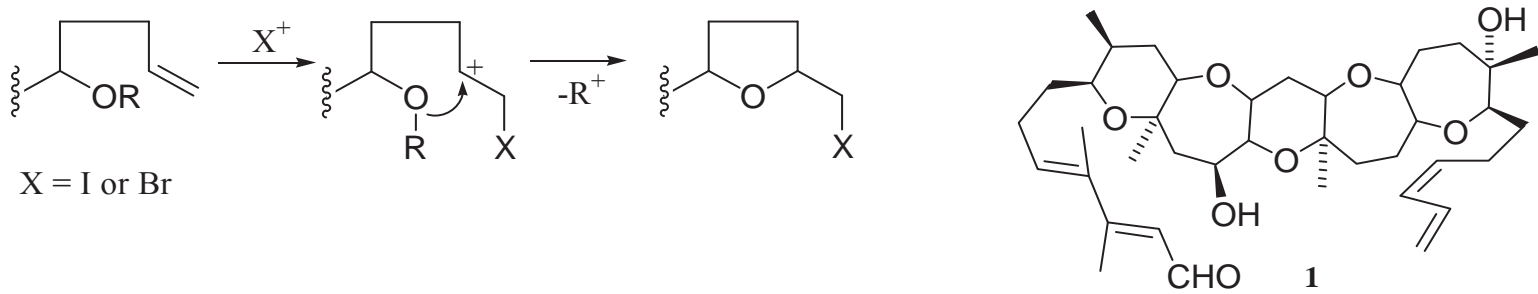

Scheme 1

Our recent research in the field of brevenal (a polyether compound isolated from laboratory cultures of the marine dinoflagellate Karenia brevis [2]) structure-activity relationships has required a synthetic method for the selective functionalization of the diene system in brevenal $\mathbf{1}$. Due to the limited availability of the natural brevenal $\mathbf{1}$, we decided to design a simpler model compound $\mathbf{2}$ to mimic the conjugated double bond system of $\mathbf{1}$. The synthesis of olefin $\mathbf{2}$ is presented in Scheme 2. The bicyclic 2 possesses two of the structural features of brevenal: a conjugated double bond in an equatorial position and a tertiary hydroxyl group. It is known that isoprenoid polycycles are used successfully as models to demonstrate the biogenetic origin of marine polyether toxins [4]. 


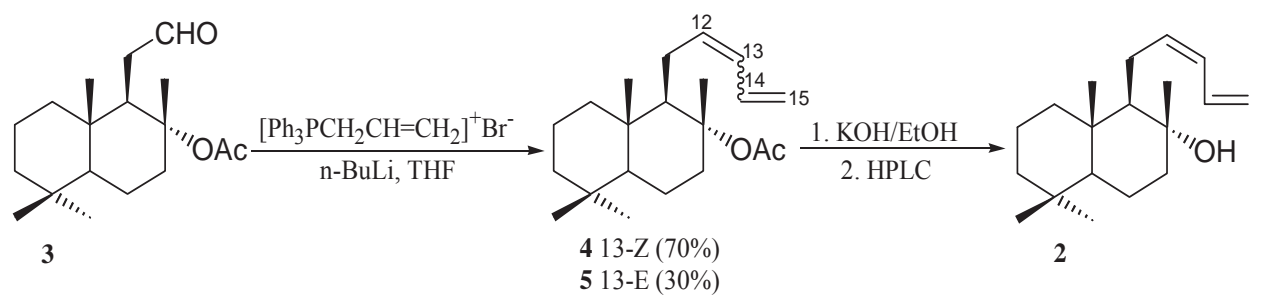

Scheme 2

The efficient functionalization of $\gamma$-pyronene using NBS has previously been described [5]. Since model compound 2 contains a similar conjugated fragment, it was expected that the reaction with NBS would lead to the addition of a bromonium ion to the more electron rich terminal double bond. The allylic stabilization of the formed carbonium ion was expected to provide a partial positive charge on the $\mathrm{C} 12$ carbon atom, which could then be cyclized efficiently through the hydroxyl group from the $\gamma$-position. This hypothesis was confirmed and the substrate 2 generated the allylic bromide 6 almost quantitatively (Scheme 3 ) under standard conditions (NBS, acetone-water, $0^{\circ} \mathrm{C}$ ).

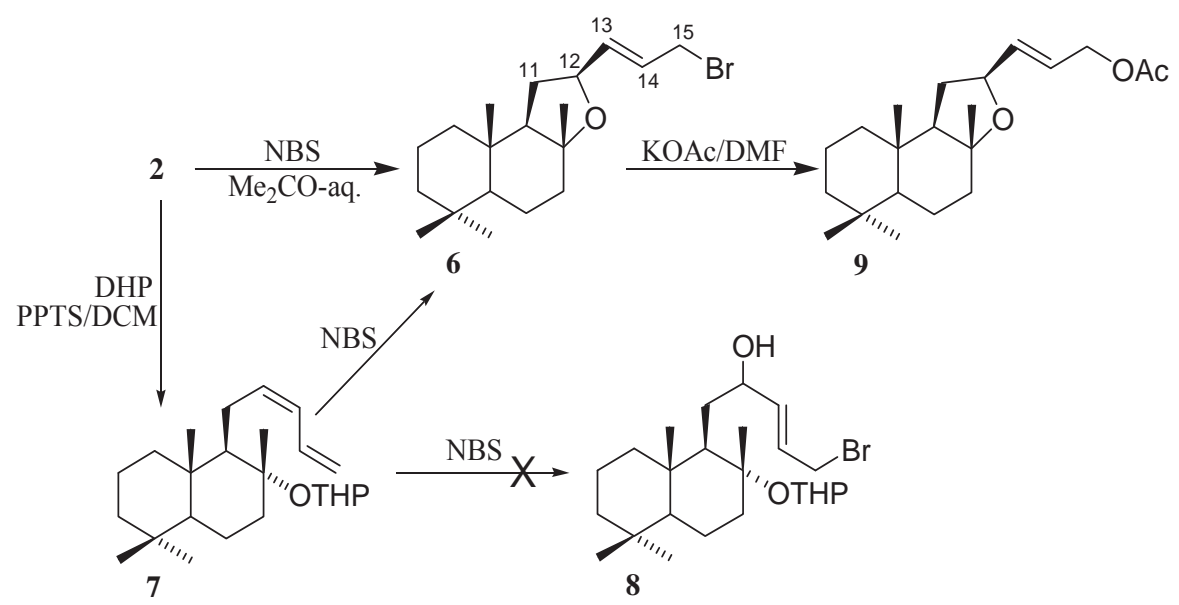

Scheme 3

The structure of $\mathbf{6}$ was elucidated on the basis of both spectroscopic data and subsequent chemical transformations. Attempts to oxidize 6 with $\mathrm{MnO}_{2}$ proved unsuccessful, which verified the absence of the secondary hydroxyl group of a hypothetical bromohydrin of type 8. It was also impossible to epoxidize $\mathbf{6}$ with $m$-CPBA under standard conditions. Such behavior normally is observed in the case of low electron density around the target double bond or as a consequence of steric hindrance. Examining the molecular models strongly supports a steric hindrance assumption. The optimized conformation of $\mathbf{6}$, presented in the figure below, shows significant shielding of the double bond by the methyl group at $\mathrm{C} 8$ and the bromine atom. This model also provides the relative stereochemistry around the $\mathrm{C} 12$ chiral center, which was confirmed by a NOESY experiment.

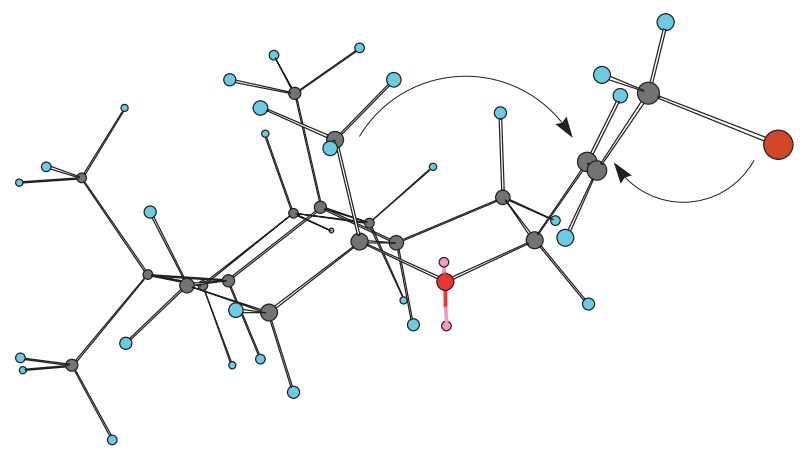

Scheme 4

Surprisingly, the same bromide 6 was obtained on the reaction of THP-protected substrate 7 with DHP [6,7]. Contrary to our expectations, the THP-protection was not stable under the reaction conditions and the reaction did not proceed with the formation of the corresponding bromohydrin $\mathbf{8}$. 
Ultimately, the utility of the allylic bromide 6 for further functionalization was checked by its transformation to the corresponding acetate $\mathbf{9}$ on treatment with potassium acetate in DMF at room temperature [8].

In summary, an alternative method for the syntheses of tetrahydrofuran cyclic compounds has been demonstrated. The procedure, based on a tandem conjugate addition - cyclization reaction provides the desired heterocyclic compound, having a lateral chain for further functionalization, with excellent yield. The practical utility and biological activity of the newly synthesized compounds is currently under investigation.

Acknowledgements: V.K acknowledges CRDF-MRDA for a travel grant MTFP-04-05.

\section{References}

[1]. Westley, J. W., Ed.; Polyether Antibiotics; Marcel Dekker: New York, 1982; Vols 1-2.

[2]. Elliott, M.C.; Williams, E. J.Chem. Soc., Perkin Trans. 1, 2001, 2303 and previous reviews in this series.

[3]. Bourdelais, A.J.; Jacocks, H.M.; Wright, J.L.C.; Bigwarfe, P.M.Jr.; Baden, D.G. J. Nat. Prod. 2005, 68, 2.

[4]. Giner, J.L. J. Org. Chem. 2005, 70, 721.

[5]. Boulin, B.; Arreguy-San Miguel, B.; Delmond, B. Tetrahedron, 54, 1998, 2753.

[6]. Diene 7. The olefin 2 (27 mg, $0.1 \mathrm{mmol})$ was dissolved in $1 \mathrm{~mL} \mathrm{DCM}$ and PPTS (2 $\mathrm{mg}, 0.008 \mathrm{mmol})$ was added to this solution with stirring at r.t. After dissolution of the solid material, $45 \mu \mathrm{L}$ of DHP (41 mg, $0.5 \mathrm{mmol})$ were added to the reaction mixture and the stirring continued overnight. Usual workup gave the crude THPderivative that was submitted to FC. Elution with 5\% EtOAc in petroleum ether gave $29 \mathrm{mg}(83 \%)$ of analytically homogeneous 13 as a diastereomeric mixture. ${ }^{1} \mathrm{H}$ NMR (ppm, in $\left.\mathrm{C}_{6} \mathrm{D}_{6}\right): 0.76$ (s); 0.78 (s); 0.79 (s); 0.81 (s); 0.83 (s) - all 9H; $1.1(\mathrm{~s}) ; 1.29(\mathrm{~s})$ - all 3H; $2.00(\mathrm{~m}, 1 \mathrm{H}) ; 2.25(\mathrm{~m}, 1 \mathrm{H}) ; 2.47(\mathrm{~m}, 1 \mathrm{H}) ; 2.69(\mathrm{~m}, 1 \mathrm{H}) ; 3.3-4.1(\mathrm{~m}, 3 \mathrm{H})$; 4.85-4.95 (m, 1H); 5.07 (d, 1H, J=10Hz); 5.15-5.25 (m, 1H); 5.65-5.80 (m, 1H); $6.03(\mathrm{t}, 1 \mathrm{H}, \mathrm{J}=10 \mathrm{~Hz}) ; 6.88-7.0$ (m, 1H). IR (cm-1): 1022; 1128; 1389; 1456; 2941.

[7]. Bromide 6. To the solution of of the protected olefin $7(28 \mathrm{mg}, 0.077 \mathrm{mmol})$ in $1.2 \mathrm{~mL}$ acetone and $0.2 \mathrm{~mL}$ water, NBS (16.5 mg, $0.093 \mathrm{mmol})$ was added in one portion at $0^{\circ} \mathrm{C}$. After $45 \mathrm{~min}$. of stirring TLC showed complete consumption of starting material. Usual workup gave $27 \mathrm{mg}$ of crude $\mathbf{6}$, homogeneous by TLC. ${ }^{1} \mathrm{H} \mathrm{NMR}(\mathrm{ppm})$ : $0.68(\mathrm{~s}, 3 \mathrm{H}) ; 0.77$ (s, 3H); 0.81 (s, 3H); 1.05 (s, 3H); 1.55 (s. 3H); 1.93/1.95 (dt, 1H); 3.56 (d, 2H); 4.29 (q, $1 \mathrm{H})$; 5.59/5.62 (dd, 1H); 5.75-5.815 (m, 1H). ${ }^{13} \mathrm{CNMR}(\mathrm{ppm}): 16.09 ; 19.14 ; 21.43 ; 21.56 ; 25.02 ; 30.23 ; 32.84 ; 33.52$; $34.01 ; 36.77 ; 40.49 ; 41.18 ; 43.06 ; 57.50 ; 61.38 ; 77.74 ; 81.27 ; 126.09 ; 139.44$. IR (cm-1): 735; 980; 1122; 1203; $1379 ; 1456 ; 1716 ; 2927$.

[8]. Acetate 9. The bromide $6(73 \mathrm{mg}, 0.17 \mathrm{mmol})$ was dissolved in $5.4 \mathrm{~mL}$ DMF and treated with freshly dried potassium acetate $(324 \mathrm{mg}, 3.3 \mathrm{mmol})$. Stirring the reaction mixture overnight at r.t. and usual workup gave the crude product. Elution with $15 \%$ EtOAc in petroleum ether gave $54 \mathrm{mg}(95 \%)$ of analytically homogeneous $9 .{ }^{1} \mathrm{H}$ NMR (ppm): 0.83 (s, 3H); 0.85 (s, 3H); 0.88 (s, 3H); 1.14 (s, 3H); 2.06 (s. 3H); 1.93/1.95 (dt, 1H); 3.56 (d, $2 \mathrm{H})$; $4.29(\mathrm{q}, 1 \mathrm{H}) ; 5.59 / 5.62(\mathrm{dd}, 1 \mathrm{H}) ; 5.75-5.815(\mathrm{~m}, 1 \mathrm{H}) .{ }^{13} \mathrm{C}$ NMR $(\mathrm{ppm}): 15.73 ; 18.59 ; 21.00 ; 21.17 ; 21.25 ; 24.65$; $29.99 ; 33.29 ; 33.73 ; 36.51 ; 40.22 ; 40.44 ; 42.62 ; 57.29 ; 61.08 ; 64.62 ; 78.09 ; 81.22 ; 124.35 ; 138.02 ; 170.99$. IR (cm-1): 968; 1026; 1122; 1228; 1379; 1458; 1741; 2937. 